\title{
High-pitch coronary CT angiography with third generation dual-source CT: limits of heart rate
}

\author{
Gordic, Sonja ; Husarik, Daniela B ; Desbiolles, Lotus ; Leschka, Sebastian ; Frauenfelder, Thomas ;
} Alkadhi, Hatem

\begin{abstract}
To determine the average heart rate (HR) and heart rate variability (HRV) required for diagnostic imaging of the coronary arteries in patients undergoing high-pitch CT-angiography (CTA) with third-generation dual-source CT. Fifty consecutive patients underwent CTA of the thoracic $(\mathrm{n}=8)$ and thoracoabdominal $(\mathrm{n}=42)$ aorta with third-generation dual-source 192-slice CT with prospective electrocardiography (ECG)-gating at a pitch of 3.2. No -blockers were administered. Motion artifacts of coronary arteries were graded on a 4-point scale. Average HR and HRV were noted. The average HR was $66 \pm 11$ beats per minute (bpm) (range 45-96 bpm); the HRV was $7.3 \pm 4.4 \mathrm{bpm}$ (range 3-20 bpm). Interobserver agreement on grade of image quality for the 642 coronary segments evaluated by both observers was good $(=0.71)$. Diagnostic image quality was found for 608 of the 642 segments $(95 \%)$ in 43 of 50 patients $(86 \%)$. In $14 \%$ of the patients, image quality was nondiagnostic for at least one segment. HR ( $\mathrm{p}=0.001)$ was significantly higher in patients with at least one non-diagnostic segment compared to those without. There was no significant difference $(\mathrm{p}>0.05)$ in HRV between patients with nondiagnostic segments and those with diagnostic images of all segments. All patients with a $\mathrm{HR}<70$ bpm had diagnostic image quality in all coronary segments. The effective radiation dose and scan time for the heart were $0.4 \pm 0.1 \mathrm{mSv}$ and $0.17 \pm 0.02 \mathrm{~s}$, respectively. Third-generation dual-source 192-slice CT allows for coronary angiography in the prospectively ECG-gated high-pitch mode with diagnostic image quality at $\mathrm{HR}$ up to $70 \mathrm{bpm}$. HRV is not significantly related to image quality of coronary CTA.
\end{abstract}

DOI: https://doi.org/10.1007/s10554-014-0445-5

Posted at the Zurich Open Repository and Archive, University of Zurich

ZORA URL: https://doi.org/10.5167/uzh-95946

Journal Article

Published Version

Originally published at:

Gordic, Sonja; Husarik, Daniela B; Desbiolles, Lotus; Leschka, Sebastian; Frauenfelder, Thomas; Alkadhi, Hatem (2014). High-pitch coronary CT angiography with third generation dual-source CT: limits of heart rate. International Journal of Cardiovascular Imaging, 30(6):1173-1179.

DOI: https://doi.org/10.1007/s10554-014-0445-5 


\title{
High-pitch coronary CT angiography with third generation dual-source CT: limits of heart rate
}

\author{
Sonja Gordic · Daniela B. Husarik • \\ Lotus Desbiolles $\cdot$ Sebastian Leschka • \\ Thomas Frauenfelder $\cdot$ Hatem Alkadhi
}

Received: 3 March 2014 / Accepted: 7 May 2014/Published online: 11 May 2014

(C) Springer Science+Business Media Dordrecht 2014

\begin{abstract}
To determine the average heart rate (HR) and heart rate variability (HRV) required for diagnostic imaging of the coronary arteries in patients undergoing highpitch CT-angiography (CTA) with third-generation dualsource CT. Fifty consecutive patients underwent CTA of the thoracic $(n=8)$ and thoracoabdominal $(n=42)$ aorta with third-generation dual-source 192 -slice CT with prospective electrocardiography (ECG)-gating at a pitch of 3.2. No $\beta$-blockers were administered. Motion artifacts of coronary arteries were graded on a 4-point scale. Average HR and HRV were noted. The average HR was $66 \pm 11$ beats per minute (bpm) (range 45-96 bpm); the HRV was $7.3 \pm 4.4 \mathrm{bpm}$ (range 3-20 bpm). Interobserver agreement on grade of image quality for the 642 coronary segments evaluated by both observers was good $(\kappa=0.71)$. Diagnostic image quality was found for 608 of the 642 segments $(95 \%)$ in 43 of 50 patients $(86 \%)$. In $14 \%$ of the patients, image quality was nondiagnostic for at least one segment. HR $(p=0.001)$ was significantly higher in patients with at least one non-diagnostic segment compared to those without. There was no significant difference $(p>0.05)$ in HRV between patients with nondiagnostic segments and those with diagnostic images of all segments. All patients with a HR $<70 \mathrm{bpm}$ had diagnostic image quality in all coronary segments. The effective radiation dose and scan time for the heart were
\end{abstract}

S. Gordic · D. B. Husarik - L. Desbiolles - S. Leschka ·

T. Frauenfelder $\cdot$ H. Alkadhi $(\bowtie)$

Institute of Diagnostic and Interventional Radiology, University

Hospital Zurich, Raemistrasse 100, 8091 Zurich, Switzerland

e-mail: hatem.alkadhi@usz.ch

L. Desbiolles · S. Leschka

Division of Radiology and Nuclear Medicine, Kantonsspital St.

Gallen, St. Gallen, Switzerland
$0.4 \pm 0.1 \mathrm{mSv}$ and $0.17 \pm 0.02 \mathrm{~s}$, respectively. Thirdgeneration dual-source 192-slice CT allows for coronary angiography in the prospectively ECG-gated high-pitch mode with diagnostic image quality at HR up to $70 \mathrm{bpm}$. $\mathrm{HRV}$ is not significantly related to image quality of coronary CTA.

Keywords Computed tomography - Coronary artery . High pitch $\cdot$ Heart rate $\cdot$ Heart rate variability

\section{Introduction}

Coronary CT angiography (CTA) has become a robust clinical tool for the diagnosis and exclusion of coronary artery disease (CAD) [1]. This is mostly due to the high sensitivity and negative predictive value of the test [2]. Recent concerns about the radiation exposure of the patient led to the introduction of low-dose protocols for cardiac imaging such as prospectively electrocardiography (ECG)gated CT in the step-and-shoot mode [2-4] and high-pitch spiral data acquisition [5-7]. Application of the latter technique is associated with a radiation dose of around $1 \mathrm{mSv}$ for cardiac CT [5-7]. High-pitch protocols, however, are not widely used because they are limited to patients with lower average heart rates (HR) and relatively low heart rate variability (HRV), precluding the wide application of the low dose technique [8]. For example Goetti et al. [9] showed that with 2nd generation dualsource CT, diagnostic image quality can be achieved only at heart rates up to 63 beats per minute (bpm) and a HRV up to $1.2 \mathrm{bpm}$.

Recently, 3rd generation dual-source CT was introduced providing a wider detector coverage acquiring 192-slices each. This CT system furthermore allows for high pitch 
values up to 3.2 , equaling a table feed of $737 \mathrm{~mm} / \mathrm{s}$. In addition, a gantry rotation time of $0.25 \mathrm{~s}$ results in a temporal resolution of $66 \mathrm{~ms}$ per image. As compared to previous 2nd generation dual-source CT-having a thinner detector (128-slices each), a smaller maximum table feed $(458 \mathrm{~mm} / \mathrm{s})$ and a lower temporal resolution $(75 \mathrm{~ms})$ these technical features of 3rd generation dual-source CT might enable prospectively ECG-gated high-pitch coronary CTA also at higher and more irregular HR.

The aim of this study was to evaluate the average HR and HRV which allows for diagnostic imaging of the coronary arteries with 3rd generation dual-source 192-slice CTA in the high-pitch mode.

\section{Subjects and methods}

\section{Study sample}

Fifty consecutive patients ( 38 men, 12 women; mean age $66 \pm 12$ years) who underwent clinically indicated thoracic $(n=8)$ or thoracoabdominal $(n=42)$ CTA were included in this study. Exclusion criteria for contrastenhanced CT were nephropathy (defined as an estimated glomerular filtration rate below $30 \mathrm{ml} / \mathrm{min} / 1.73 \mathrm{~m}^{2}$ ) and known hypersensitivity to iodinated contrast media. Patients with elevated and irregular heart rates were not excluded from the study. No premedication for heart rate control or vasodilatation was added to the patients' baseline medication.

Thoracic CTA was performed for postoperative followup after thoracic vascular surgery $(n=5)$ and preoperative evaluation before aortic valve and root surgery $(\mathrm{n}=3)$. The clinical indications for thoracoabdominal CTA included postoperative follow-up after thoracoabdominal vascular surgery or endovascular aneurysm repair $(n=26)$, evaluation of suspected aortic dissection $(n=3)$ and follow-up examination of conservatively treated aortic aneurysm $(\mathrm{n}=13)$.

Institutional review board approval was obtained. Written informed consent for this retrospective study was waived by our local ethics committee, because all CT studies were clinically indicated.

\section{CT data acquisition and image reconstruction}

All exams were performed with a 3rd generation dualsource CT system (SOMATOM Force, Siemens Healthcare, Forchheim, Germany) equipped with an integrated high-resolution detector (Stellar Technology, Siemens) [10]. The amount of contrast material was adjusted according to the body mass index of the patient and ranged between 70 and $90 \mathrm{ml}$ iopromide (Ultravist 300, $300 \mathrm{mg}$
Iodine/ml; Bayer Schering Pharma, Berlin, Germany). Contrast media was injected intravenously at a flow rate of $5 \mathrm{ml} / \mathrm{s}$ followed by $60 \mathrm{ml}$ saline solution with the same flow rate. Contrast material application was controlled by bolus tracking in the ascending aorta with a signal attenuation threshold set at $100 \mathrm{HU}$ at $120 \mathrm{kVp}$ with the formal CT data acquisition beginning with a delay of $10 \mathrm{~s}$ after this threshold was reached. CT parameters were: slice collimation of $192 \times 0.6 \mathrm{~mm}$ by means of a z-flying focal spot; gantry rotation time $250 \mathrm{~ms}$; pitch 3.2. Studies were performed with automated tube current modulation (CAREDose4D, Siemens) using a reference tube currenttime product of $130 \mathrm{mAs}$ and using automated attenuationbased tube voltage selection (CAREkV, Siemens) with a reference tube potential of $120 \mathrm{kVp}$. The level $2 \mathrm{~cm}$ below the tracheal carina was manually set as the target for image acquisition at $60 \%$ of the RR interval. The start time of CT acquisition at the most cranial position of the chest was then automatically calculated by the CT software in accordance with the ECG, in order to time the data acquisition to arrive at the indicated level (i.e. $2 \mathrm{~cm}$ below the carina) at $60 \%$ of the RR interval.

Images of the heart and coronary arteries were reconstructed with Advanced Model-based Iterative Reconstruction (ADMIRE) at a strength level of three and with a slice thickness of $0.6 \mathrm{~mm}$ and an increment of $0.4 \mathrm{~mm}$ using a medium smooth reconstruction kernel (Bv36). ADMIRE, which was introduced with 3rd generation dualsource 192-slice CT, combines a statistical data modeling in the raw data domain and a model-based noise detection in the image domain in an iterative approach. With ADMIRE, five strength levels are available for noise suppression. We chose the medium strength level of three in our study.

The average, the minimum, and the maximum heart rates during ten heartbeats before the start of CTA data acquisition were noted by one radiologist. Subsequent analyses were performed using a picture archiving and communication system (PACS) workstation on a highdefinition liquid crystal display monitor (BARCO-Medical Imaging Systems, Kortrijk, Belgium) using Impax (Version 6.4.0.4551, Agfa-Gevaert, Mortsel, Belgium).

Data readout

Motion artifacts

The coronary tree was subdivided for segment-based analysis according to the modified 16-segment scheme proposed by the American Heart Association [11]. The right coronary artery (RCA) included segments $1-4$, and segment 5 was defined as the left main coronary artery (LMA). The left anterior descending artery (LAD) included 
segments $6-10$. Segments $11-15$ comprised the circumflex artery $(\mathrm{CX})$. An intermediate artery was defined as segment 16. Because of anatomic variation, not all coronary 16 segments were present in each patient.

For determining the interobserver variability, two independent radiologists ( 3 and 7 years of experience in cardiovascular imaging) blinded to each other's findings and to HR and HRV classified image quality for each coronary segment with regard to motion artifacts using a modified 4-point grading scale as previously described [12]: A score of 1 indicated excellent image quality with no artifacts (unrestricted evaluation of the coronary arteries); a score of 2 indicated good image quality (minor artifacts, slight blurring of the vessel walls, good diagnostic quality); a score of 3 indicated adequate image quality (moderate artifacts, moderate blurring of the vessel wall, still acceptable and diagnostic); and a score of 4 indicated non-diagnostic image quality (severe artifacts and vessel wall blurring impairing evaluation).

\section{Objective image quality}

Attenuation and image noise were assessed as objective determinants of image quality by one of the two readers who performed the subjective image quality readout. Attenuation in the ascending aorta at the level of the left main artery was measured with a region of interest (ROI) (average size $200 \mathrm{~mm}^{2}$ ) avoiding calcifications and plaques. Image noise was determined as the standard deviation (SD) of the attenuation in the ROI.

\section{Estimation of radiation dose}

The effective radiation doses were calculated with a method proposed by the European Working Group for Guidelines on Quality Criteria for CT [13] by using the dose-length product and a conversion coefficient $(k)$. For thoracic and coronary CTA a conversion coefficient of $0.014 \mathrm{mSv} / \mathrm{mGycm}$ was used. For thoracoabdominal CTA the mean of the region-specific conversion coefficients ( $k=0.016 \mathrm{mSv} / \mathrm{mGycm})$ of the chest $(k=0.014 \mathrm{mSv} /$ $\mathrm{mGycm})$, abdomen $(k=0.015 \mathrm{mSv} / \mathrm{mGycm})$, and pelvis ( $k=0.019 \mathrm{mSv} / \mathrm{mGycm}$ ) was used, as previously shown [14]. The dose-length product was obtained from the electronically logged patient protocol. In addition, the scan length of the heart was measured in z-axis in each patient. By multiplying the $\mathrm{CTDI}_{\mathrm{vol}}$ by the scan length of the heart and the conversion factor for the chest, the effective radiation dose for the heart was estimated.

Statistical analysis

Quantitative parameters were expressed as means \pm standard deviations for normally distributed and medians \pm interquartile

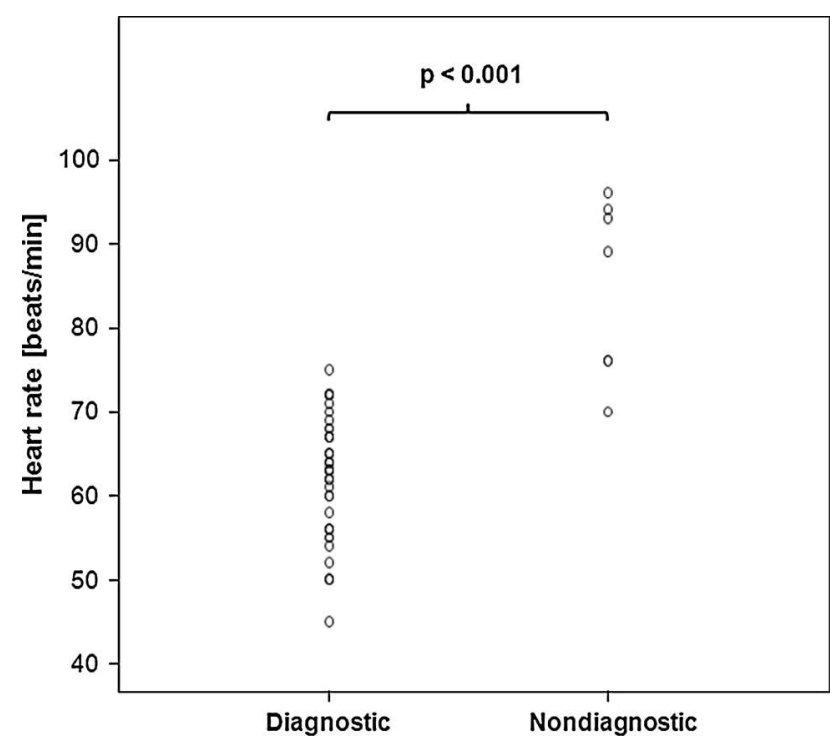

Fig. 1 Dot plot representing the relationship between HR and patients with at least one nondiagnostic coronary segment and those without nondiagnostic segments in high-pitch CTA

ranges for non-normally distributed variables. Categorical variables were expressed as frequencies or percentages. Normality was tested using the Kolmogorov-Smirnov test.

Interobserver agreement on image quality of the coronary artery segments was evaluated with Cohen's kappa statistics. A kappa value of more than 0.80 was interpreted as excellent interobserver agreement; 0.61-0.80, good; $0.41-0.60$, moderate; $0.21-0.40$, fair; and less than 0.20 , poor.

HRV was defined as the difference between minimum and maximum heart rate in the 10 heartbeats before imaging acquisition. The median of the HR and the median of HRV were calculated and used to subdivide the study sample into four groups: low HR with low HRV, low HR with high HRV, high HR with low HRV and high HR with high HRV.

The $t$ test for independent samples was used to analyze differences in average HR, HRV and image noise between patients with diagnostic image quality of all coronary segments and those with at least one segment with motion artifacts (Fig. 1).

Receiver operating characteristics (ROC) analyses for the prediction of nondiagnostic studies were performed for HR and HRV. Point estimates, $95 \%$ CIs, and the area under the curve (AUC) were calculated. AUCs were compared using the $\mathrm{Z}$ test.

All statistical analyses were conducted using commercially available software (SPSS, release 21.0; SPSS, Chicago, IL, USA). A two-tailed $p$ value $<0.05$ was considered to indicate statistical significance (Fig. 2). 


\section{Results}

The average HR was $66 \pm 11 \mathrm{bpm}$ (range $45-96 \mathrm{bpm}$ ), and the average HRV before and during image acquisition was $7.3 \pm 4.4 \mathrm{bpm}$ (range $3-20 \mathrm{bpm}$ ), (Table 1). The average scan length of the heart was $124 \pm 12 \mathrm{~mm}$ (scan time $0.17 \pm 0.02 \mathrm{~s}$ ); the average scan length for thoracic CTA was $369 \pm 21 \mathrm{~mm}$ (scan time, $0.54 \pm 0.08 \mathrm{~s}$ ); and

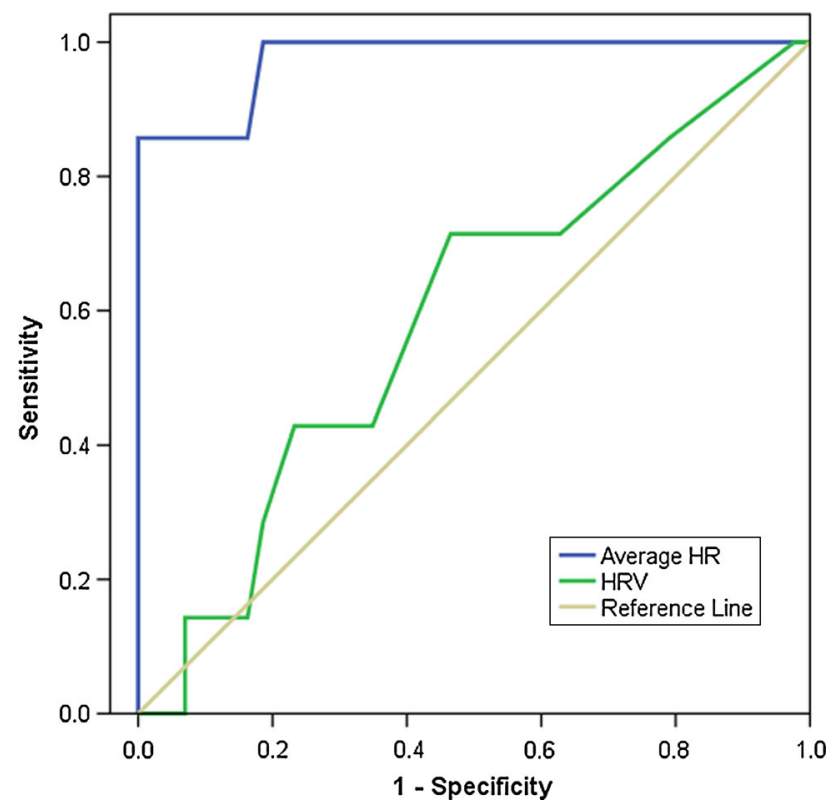

Fig. 2 Receiver operating characteristic (ROC) analyses for average heart rate (HR) and HR variability (HRV) in relation to image quality of high-pitch coronary CT angiography. Area under the curve for HR $(\mathrm{AUC}=0.98)$ is significantly higher than that for $\mathrm{HRV}(\mathrm{AUC}=0.59$; $p<0.01)$ the average scan length for thoracoabdominal CTA was $666 \pm 57 \mathrm{~mm}$ (scan time, $1.0 \pm 0.03 \mathrm{~s}$ ).

\section{Motion artifacts}

A total of 642 coronary artery segments were evaluated by both observers. The interobserver agreement for image quality grading was good $(\kappa=0.711)$. Image quality was rated as excellent (score 1) for $87 \%(561 / 642)$ of segments, good (score 2) for $6 \%(35 / 642)$, moderate (score 3) for $2 \%(12 / 642)$, and nondiagnostic for $5 \%(34 / 642)$. Figures 3 and 4 show examples of images with different quality scores. In 43 patients ( $86 \%$ ) all coronary artery segments were visualized with diagnostic image quality (scores 1, 2 and 3), and nondiagnostic image quality was found in seven patients (14\%). Nondiagnostic image quality was most often found in the RCA $50 \%(17 / 34)$ in the following distribution: proximal $\mathrm{RCA}(\mathrm{n}=5)$, mid RCA $(n=5)$, distal RCA $(n=4)$, and posterior descending artery $(n=3)$. Nondiagnostic image quality was present less frequently in the LAD $38 \%(\mathrm{n}=13)$ and CX $12 \%(\mathrm{n}=4)$.

\section{Objective image quality}

The average image noise in the ascending aorta was $38.3 \pm 10.3$ HU. Image noise was not significantly different in patients with at least one nondiagnostic segment $(39.4 \pm 9.7 \mathrm{HU})$ as compared to those without a nondiagnostic coronary segment $(38.2 \pm 10.6 \mathrm{HU}, p=0.56)$.

The average attenuation in the ascending aorta was $383.5 \pm 115.4 \mathrm{HU}$. Attenuation was not significantly different in patients with at least one nondiagnostic segment
Table 1 Heart rate and image quality in groups separated by median average heart rate (HR) and median heart rate variability (HRV)

\begin{tabular}{lllll}
\hline Finding & $\begin{array}{l}\text { Low HR, } \\
\text { low HRV }\end{array}$ & $\begin{array}{l}\text { Low HR, } \\
\text { high HRV }\end{array}$ & $\begin{array}{l}\text { High HR, } \\
\text { low HRV }\end{array}$ & $\begin{array}{l}\text { High HR, } \\
\text { high HRV }\end{array}$ \\
\hline $\begin{array}{l}\text { Number of patients } \\
\text { Heart rate parameter }\end{array}$ & 17 & 11 & 8 & 14 \\
$\quad$ HR (bpm) & $59 \pm 5$ & $58 \pm 7$ & $76 \pm 10$ & $74 \pm 8$ \\
Range (bpm) & $50-64$ & $45-64$ & $65-94$ & $67-96$ \\
HRV (bpm) & $3.7 \pm 1.0$ & $10.0 \pm 4.0$ & $4.5 \pm 1.0$ & $11 \pm 3.8$ \\
$\begin{array}{l}\text { Image quality per segment } \\
\text { Score 1 }\end{array}$ & $97.3(214 / 220)$ & $96.5(138 / 143)$ & $78.8(82 / 104)$ & $72.5(127 / 175)$ \\
$\begin{array}{l}\text { Score 2 } \\
\text { Score 3 }\end{array}$ & $2.7(6 / 220)$ & $3.5(5 / 143)$ & $5.8(6 / 104)$ & $10.3(18 / 175)$ \\
Score 4 & - & - & $2.9(3 / 104)$ & $5.2(9 / 175)$ \\
Image quality per patient & - & - & $12.5(13 / 104)$ & $12.0(21 / 175)$ \\
All segments have & & & & $64(9 / 14)$ \\
$\quad \begin{array}{l}\text { diagnostic image quality } \\
(\%)\end{array}$ & $100(17 / 17)$ & $100(11 / 11)$ & $75(6 / 8)$ & \\
$\begin{array}{l}\text { Not all segments have } \\
\text { diagnostic image quality } \\
(\%)\end{array}$ & - & & & $36(5 / 14)$
\end{tabular}



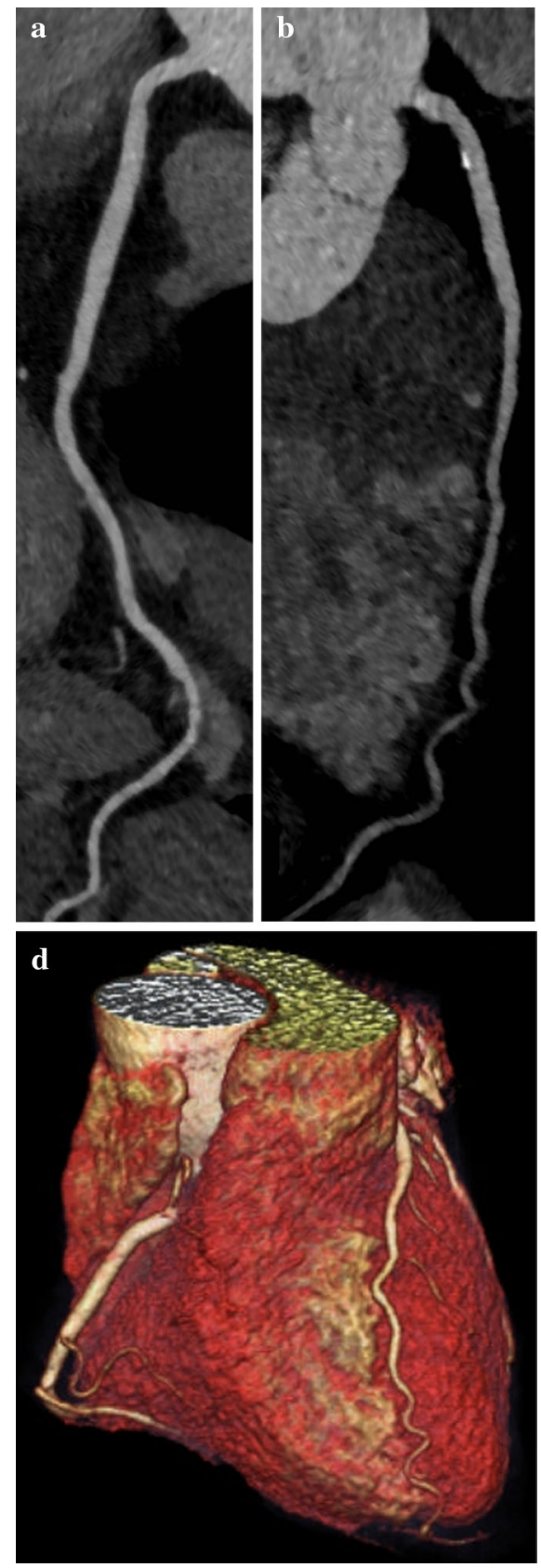

Fig. 3 68-year-old man with an average HR of $70 \mathrm{bpm}$ and a HRV of $6 \mathrm{bpm}$ undergoing CT for postoperative follow-up after aortic dissection. Curved multiplanar reformations of the right coronary (a), left anterior descending (b), and circumflex (c) artery and volume-rendered image (d) showing excellent image quality (score 1) of all segments

$(405.5 \pm 160.0 \mathrm{HU})$ and those without nondiagnostic segments $(380.0 \pm 108.5 \mathrm{HU}, p=0.59)$.

Influence of average HR and HRV on image quality

Patients with nondiagnostic segments had a significantly $(p<0.001)$ higher average HR $(85 \pm 11 \mathrm{bpm})$ than those

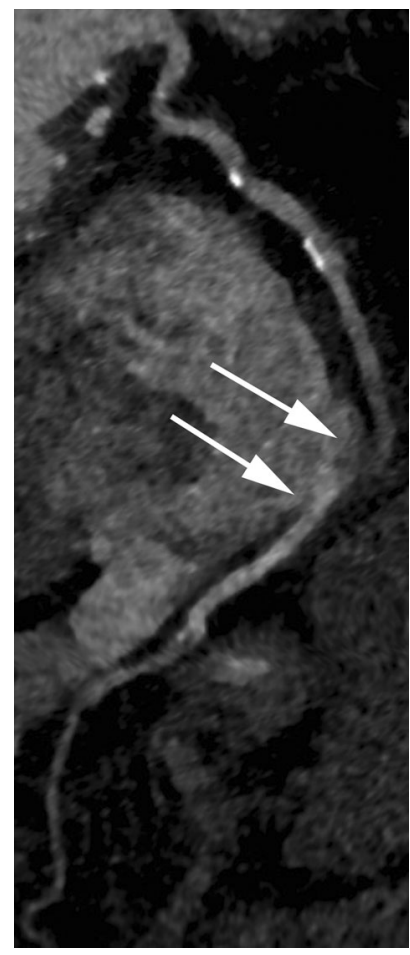

Fig. 4 70-year-old man with an average HR of $76 \mathrm{bpm}$ and a HRV of $7 \mathrm{bpm}$ undergoing CT for follow-up of conservatively treated aortic aneurysm. Curved multiplanar reformation of the right coronary artery shows nondiagnostic image quality of the middle segment

with diagnostic images of all coronary artery segments (62 $\pm 7 \mathrm{bpm})$ (Fig. 1). There was no significant difference ( $p=0.576$ ) between the HRV of patients with nondiagnostic segments $(8.2 \pm 4.2 \mathrm{bpm})$ and those with diagnostic images of all segments $(7.1 \pm 4.5 \mathrm{bpm})$. In our study sample, the median HR was $65 \mathrm{bpm}$, and the median HRV was $6.3 \mathrm{bpm}$. When the data on patients were distributed into four groups separated by these thresholds, all coronary artery segments were visualized with diagnostic image quality in patients with a low heart rate (average HR, $<65 \mathrm{bpm}$ ) independent of the HRV (Table 1). In contrast, CT studies with nondiagnostic visualization of the coronary arteries most frequently occurred in the evaluation of patients with both a high $\mathrm{HR}$ and high HRV $(36 \%, 5 / 14)$. In patients with a high heart rate ( $>65 \mathrm{bpm})$ the lowest HRV associated with nondiagnostic segments was 3 bpm (HR 93 bpm).

ROC analysis showed the average HR to be significantly related to coronary CTA image quality $(p<0.001$; $\mathrm{AUC}=0.975 \pm 0.03 ; 95 \%$ CI 0.925-1.00), with an HR frequency $<70 \mathrm{bpm}$ as the optimal threshold to distinguish between diagnostic and nondiagnostic studies. At $\mathrm{HR}<70 \mathrm{bpm}$ all CTA studies were diagnostic. At $\mathrm{HR} \geq 70$ to $<75 \mathrm{bpm}$ studies were diagnostic in $87.5 \%$ and at $\mathrm{HR} \geq 75 \mathrm{bpm}$ in $14.3 \%$ of the cases (Table 1).

HRV $\quad(p=0.44 ; \quad$ AUC $=0.59 \pm 0.12 ; \quad 90 \% \quad$ CI $0.37-0.82$ ) was not significantly related to image quality of 
coronary CTA (Fig. 2). AUC of HRV was significantly $(p<0.01)$ smaller than that of HR.

\section{Estimation of radiation dose}

The mean effective radiation dose of the heart was $0.4 \pm 0.1 \mathrm{mSv}$, the mean dose for thoracic CTA was $1.2 \pm 0.2 \mathrm{mSv}$, and the mean dose for thoracoabdominal CTA was $3.1 \pm 1.3 \mathrm{mSv}$.

\section{Discussion}

Our study indicates that high-pitch CTA performed with a 3rd generation 192-slice dual-source CT system yields diagnostic image quality of the coronary arteries in patients with an average HR up to $70 \mathrm{bpm}$. At HR between 70 and $75 \mathrm{bpm}$, studies were diagnostic in $88 \%$, and at $\mathrm{HR} \geq 75$ bpm image quality was diagnostic in only $14 \%$ of patients. In contrast to the average HR, HRV was not significantly related to the image quality of the coronary arteries. The high-pitch data acquisition of the heart is fast, taking less than $0.2 \mathrm{~s}$, and is associated with a low radiation dose of $0.4 \mathrm{mSv}$.

The average HR is a well known factor affecting the image quality of cardiac CT. This holds true also for highpitch coronary CTA [5-9, 15, 16]. Using 2nd generation dual-source CT, Goetti et al. [9] reported a diagnostic depiction of the coronary arteries for patients with an average HR $<63 \mathrm{bpm}$. Using a similar CT system, Scharf et al. [8] show that all patients with an average $\mathrm{HR}<64 \mathrm{bpm}$ had a diagnostic image quality of all coronary segments evaluated by high-pitch CTA. In our study evaluating this issue with 3 rd generation dual-source CT, patients with an average HR up to $70 \mathrm{bpm}$ had a diagnostic image quality of all their coronary segments. Differences to the studies mentioned above are most likely the differences in detector width (192- vs. 128-slice) and hence, table feed ( 737 vs. $458 \mathrm{~mm} / \mathrm{s}$ ), as well the differences in gantry rotation time ( 0.28 vs. $0.25 \mathrm{~s})$ and hence, temporal resolution per image (75 vs. $66 \mathrm{~ms}$ ).

Similar to the average HR, HRV is another factor known to influence the image quality of coronary CTA. This holds particularly true for the high-pitch mode, where HRV is considered a major determinant for the prediction of the data acquisition window. Previous studies with 2 nd generation dual-source CT showed that-when the heart rate is irregular prior to CT data acquisition, the image quality is degraded because the window in diastole is not accurately targeted [8, 9, 15]. Goetti et al. [9] reported a diagnostic depiction of the coronary arteries for patients with a HRV below $1.2 \mathrm{bpm}$. Similarly, Scharf et al. [8] reported that all patients with a HRV below $1.3 \mathrm{bpm}$ had a diagnostic image quality of all coronary segments evaluated with 2nd generation dual-source CT. In distinction, our study indicates the HRV (being similarly defined as in the above mentioned studies) was not significantly related to the image quality of the coronary arteries in high-pitch coronary CTA using 3rd generation dual-source CT.

In case of a high HRV, it is likely that the prospectively ECG-gated high-pitch CT scan occurs in systole instead of the diastole. With increasing HR, the phase of diastasis progressively disappears, and the smallest coronary velocity in systole approaches that in diastole. Given these diminished differences in coronary velocity between diastole and systole, it appears that the time-point of image reconstruction within the cardiac cycle becomes less relevant with 3 rd generation dual-source $\mathrm{CT}$, owing to the shorter time window for image acquisition $(170 \mathrm{~ms}$ for an average cranio-caudal scan length of $12.4 \mathrm{~cm}$ ) in combination with the high temporal resolution.

Several methods have been developed for reducing the radiation dose of coronary CTA [17]. These include prospective ECG-gating in the step-and-shoot mode [2-4], automated exposure control [18, 19], high-pitch spiral acquisition [5-9, 15, 16], and use of the noise reducing properties with iterative reconstruction algorithms $[4,7$, 19-21]. Recent studies using a combination of these techniques allowed for coronary CT angiography at low radiation doses. For example Hou et al. [4] reported an effective radiation dose of $1.2 \mathrm{mSv}$ using iterative reconstruction in prospectively ECG-gated 256-slice coronary CTA. Chen et al. [19] reported an effective radiation dose of $0.9 \mathrm{mSv}$ using a second-generation 320-slice wide volume coverage CT with iterative reconstruction and automated exposure control. Recently, Yin et al. [7] reported a radiation dose of $0.6 \mathrm{mSv}$ using 2 nd generation dualsource CT and prospectively ECG-gated high-pitch spiral acquisition, whereby the tube current-time product was set to $50 \%$ of the standard value and images were reconstructed with sinogram-affirmed iterative reconstruction. In our study, the effective radiation dose for the heart was $0.4 \mathrm{mSv}$ using a combination of the techniques described above. It is important to know, however, that the current CT protocol was optimized for CTA of the thoracic and thoracoabdominal aorta and not exclusively for the heart. Thus, the lowest possible radiation dose for coronary CTA with 3rd generation dual-source $\mathrm{CT}$ remains to be determined.

Our study suffers from some limitations: First, we used a qualitative image quality scoring system that might have been influence by subjectivity bias. However, the kappa value of 0.71 indicates a good interobserver agreement. Second, CT was not performed for the assessment of coronary artery stenosis, and no results of cardiac catheterization studies were available for comparison. Therefore, 
the diagnostic performance of coronary CTA in the highpitch acquisition mode for the detection of coronary artery disease was not assessed, and we did not prove our scoring threshold of acceptable image quality for diagnostic purposes.

In conclusion, our study demonstrates that 3rd generation dual-source 192-CT in the high pitch mode allows for coronary imaging with diagnostic image quality in patients with a HR up to $70 \mathrm{bpm}$ regardless of $\mathrm{HRV}$ at a low radiation dose.

Conflict of interest None.

\section{References}

1. Taylor AJ, Cerqueira M, Hodgson JM et al (2010) ACCF/SCCT/ ACR/AHA/ASE/ASNC/NASCI/SCAI/SCMR 2010 appropriate use criteria for cardiac computed tomography. A report of the American College of Cardiology Foundation appropriate use criteria task force, the Society of Cardiovascular Computed Tomography, the American College of Radiology, the American Heart Association, the American Society of Echocardiography, the American Society of Nuclear Cardiology, the North American Society for Cardiovascular Imaging, the Society for Cardiovascular Angiography and Interventions, and the Society for Cardiovascular Magnetic Resonance. J Cardiovasc Comput Tomogr 4:407 e1-407 e33

2. von Ballmoos MW, Haring B, Juillerat P, Alkadhi H (2011) Meta-analysis: diagnostic performance of low-radiation-dose coronary computed tomography angiography. Ann Intern Med 154:413-420

3. Hsieh J, Londt J, Vass M, Li J, Tang X, Okerlund D (2006) Stepand-shoot data acquisition and reconstruction for cardiac X-ray computed tomography. Med Phys 33:4236-4248

4. Hou Y, Zheng J, Wang Y, Yu M, Vembar M, Guo Q (2013) Optimizing radiation dose levels in prospectively electrocardiogram-triggered coronary computed tomography angiography using iterative reconstruction techniques: a phantom and patient study. PLoS One 8:e56295

5. Achenbach S, Goroll T, Seltmann M et al (2011) Detection of coronary artery stenoses by low-dose, prospectively ECG-triggered, high-pitch spiral coronary CT angiography. JACC Cardiovasc Imaging 4:328-337

6. Achenbach S, Marwan M, Schepis T et al (2009) High-pitch spiral acquisition: a new scan mode for coronary CT angiography. J Cardiovasc Comput Tomogr 3:117-121

7. Yin WH, Lu B, Hou ZH et al (2013) Detection of coronary artery stenosis with sub-milliSievert radiation dose by prospectively ECG-triggered high-pitch spiral CT angiography and iterative reconstruction. Eur Radiol 23:2927-2933
8. Scharf M, Bink R, May MS et al (2011) High-pitch thoracic CT with simultaneous assessment of coronary arteries: effect of heart rate and heart rate variability on image quality and diagnostic accuracy. JACC Cardiovasc Imaging 4:602-609

9. Goetti R, Baumuller S, Feuchtner G et al (2010) High-pitch dualsource CT angiography of the thoracic and abdominal aorta: is simultaneous coronary artery assessment possible? AJR Am J Roentgenol 194:938-944

10. Morsbach F, Desbiolles L, Plass A et al (2013) Stenosis quantification in coronary CT angiography: impact of an integrated circuit detector with iterative reconstruction. Invest Radiol 48:32-40

11. Austen WG, Edwards JE, Frye RL et al (1975) A reporting system on patients evaluated for coronary artery disease. Report of the Ad Hoc Committee for Grading of Coronary Artery Disease, Council on Cardiovascular Surgery, American Heart Association. Circulation 51(4 Suppl):5-40

12. Leschka S, Stolzmann P, Desbiolles L et al (2009) Diagnostic accuracy of high-pitch dual-source CT for the assessment of coronary stenoses: first experience. Eur Radiol 19:2896-2903

13. Menzel HG, Harrison JD (2012) Doses from radiation exposure. Ann ICRP 41(3-4):12-23

14. Macari M, Chandarana H, Schmidt B, Lee J, Lamparello P, Babb J (2006) Abdominal aortic aneurysm: can the arterial phase at CT evaluation after endovascular repair be eliminated to reduce radiation dose? Radiology 241:908-914

15. Lell M, Hinkmann F, Anders K et al (2009) High-pitch electrocardiogram-triggered computed tomography of the chest: initial results. Invest Radiol 44:728-733

16. Stolzmann P, Goetti RP, Maurovich-Horvat P et al (2011) Predictors of image quality in high-pitch coronary CT angiography. AJR Am J Roentgenol 197:851-858

17. Halliburton SS, Abbara S, Chen MY et al (2011) SCCT guidelines on radiation dose and dose-optimization strategies in cardiovascular CT. J Cardiovasc Comput Tomogr 5:198-224

18. Deetjen A, Mollmann S, Conradi G et al (2007) Use of automatic exposure control in multislice computed tomography of the coronaries: comparison of 16-slice and 64-slice scanner data with conventional coronary angiography. Heart 93:1040-1043

19. Chen MY, Shanbhag SM, Arai AE (2013) Submillisievert median radiation dose for coronary angiography with a second-generation 320-detector row CT scanner in 107 consecutive patients. Radiology 267:76-85

20. Gosling O, Loader R, Venables P et al (2010) A comparison of radiation doses between state-of-the-art multislice CT coronary angiography with iterative reconstruction, multislice CT coronary angiography with standard filtered back-projection and invasive diagnostic coronary angiography. Heart 96:922-926

21. Leipsic J, Labounty TM, Heilbron B et al (2010) Estimated radiation dose reduction using adaptive statistical iterative reconstruction in coronary CT angiography: the ERASIR study. AJR Am J Roentgenol 195:655-660 